\title{
Partition regularity with congruence conditions
}

\author{
Ben BARBER AND IMRE LEADER
}

\begin{abstract}
An infinite integer matrix $A$ is called image partition regular if, whenever the natural numbers are finitely coloured, there is an integer vector $x$ such that $A x$ is monochromatic. Given an image partition regular matrix $A$, can we also insist that each variable $x_{i}$ is a multiple of some given $d_{i}$ ? This is a question of Hindman, Leader and Strauss.

Our aim in this short note is to show that the answer is negative. As an application, we disprove a conjectured equivalence between the two main forms of partition regularity, namely image partition regularity and kernel partition regularity.
\end{abstract}

AMS 2000 SUbJECT ClASSifications: Primary 05D10; secondary 03E02. KEYWORDS AND PHRASES: Partition regular systems, Ramsey theory.

\section{Introduction}

We call an integer matrix $A$ (possibly infinite but with only finitely many non-zero entries in each row) image partition regular if, whenever $\mathbb{N}$ is finitely coloured, there is a vector $x$ with integer entries such that every entry of $A x$ is in $\mathbb{N}$ and has the same colour (" $A x$ is monochromatic"). For example, Schur's theorem states that whenever we finitely colour the natural numbers we can find $x$ and $y$ such that $x, y$ and $x+y$ are all the same colour: that is, the matrix

$$
\left(\begin{array}{ll}
1 & 0 \\
0 & 1 \\
1 & 1
\end{array}\right)
$$

is image partition regular. ${ }^{1}$

In the finite case, partition regularity is very well understood (see [5, 4] and, for a general overview, [2]). In contrast, in the infinite case the situation is far less clear, and several very basic questions remain unanswered-see

arXiv: 1304.5173

${ }^{1}$ What we call 'image partition regular' is sometimes called 'image partition regular over $\mathbb{Z}$ ' (or 'weakly image partition regular') to indicate that the variables take values in $\mathbb{Z}$. 
the survey [3]. Our aim in this note is to give negative answers to three questions from [3].

Question 1 ([3, Q8]). Let $A$ be an image partition regular matrix and $\left(d_{i}\right)$ be a sequence of integers. Is it true that, whenever the natural numbers are finitely coloured, there is a monochromatic image Ax such that the variables $x_{i}$ satisfy $x_{i} \equiv 0\left(\bmod d_{i}\right)$ ?

[We remark that the question in [3] does not take quite this form. It is phrased in terms of 'kernel partition regularity' and is restricted to systems for which the variables can always be taken to have the same colour as $A x$. Our counterexample for Question 1 will also be a counterexample for this more specialised question.]

Note that of course we cannot ask for $x_{i} \equiv a_{i}\left(\bmod d_{i}\right)$ for arbitrary $a_{i}$. For example, in Schur's theorem we cannot ask for $x$ to be odd; for then colouring the odd numbers red and the even numbers blue forces all of $x, y$ and $x+y$ to be odd.

A related notion is that of kernel partition regularity. We call a matrix A kernel partition regular if, whenever the natural numbers are finitely coloured, there is a monochromatic vector $x$ such that $A x=0$. It is natural to ask whether the two notions of image and kernel partition regularity are 'essentially the same', in a sense we now describe.

Given a matrix $A$ (with only finitely many non-zero entries in each row) we define a matrix $B(A)$, expressing the linear dependences between the rows of $A$, as follows. Let $\left\{r_{i}: i \in I\right\}$ be a maximal linearly independent set of rows of $A$, and write each of the remaining rows $\left\{s_{j}: j \in J\right\}$ as a linear combination of the $r_{i}$. Let $B(A)$ be the matrix corresponding to these linear equations. That is, for each $j \in J$ write $s_{j}=\sum_{i \in I} c_{j i} r_{i}$, and let $B(A)$ be the $J \times(I \cup J)$ matrix with left-hand side $\left(c_{j i}\right)$ and right-hand side -1 times the $J \times J$ identity matrix. ${ }^{2}$

If $A$ is image partition regular, then certainly $B(A)$ is kernel partition regular, because any monochromatic image of $A$ is in the kernel of $B(A)$. Conversely:

Question 2 ([3, Q7]). If $B(A)$ is kernel partition regular, must $A$ be image partition regular?

Finally, we say that an integer matrix $A$ (with only finitely many nonzero entries in each row) is image partition regular over $\mathbb{Q}$ if, whenever

\footnotetext{
${ }^{2}$ The reader may be worried about the case when the rows of $A$ are linearly independent. In that case $B(A)$ is the empty matrix, which is automatically kernel partition regular.
} 
$\mathbb{Q} \backslash\{0\}$ is finitely coloured, there is a vector $x$ with entries in $\mathbb{Q}$ such that $A x$ is monochromatic.

Question 3 ([3, Q9]). If $A$ is image partition regular over $\mathbb{Q}$, must it be image partition regular? in [1].

The corresponding question for kernel partition regularity was answered

Our aim in this short note is to show that the answer to each of these questions is negative. The three questions turn out to be related: once we have a counterexample for Question 1, counterexamples for Question 2 and Question 3 will follow.

\section{The counterexample}

We seek a matrix $A$, and a sequence of natural numbers $\left(d_{i}\right)$, such that $A$ is image partition regular, but we cannot always find a monochromatic image $A x$ satisfying $x_{i} \equiv 0\left(\bmod d_{i}\right)$ for each $i$. The matrix we shall make use of comes from a recent result of Barber, Hindman and Leader [1]. It is simpler to give the system of expressions corresponding to its rows than to describe the image partition regular matrix explicitly. In the following system, the variables are $y$ and $x_{i j}, 1 \leq j \leq i$.

Theorem 4 ([1, Theorem 15]). Let $\left(c_{n}\right)$ be any sequence of integer coefficients. Then the system of expressions

$$
\begin{array}{rcc}
x_{11}+c_{1} y & x_{11} & y \\
x_{21}+x_{22}+c_{2} y & x_{21} & \\
x_{31}+x_{32}+x_{33}+c_{3} y & x_{22} &
\end{array}
$$

is image partition regular.

[The result in [1] was stated for $c_{n}=2^{n}$, but as remarked in [1], the proof works for any integer sequence $\left(c_{n}\right)$.]

Roughly speaking, our idea is to 'simulate' the condition that a variable be odd by giving some conditions on the other variables. This will be achieved by a particular choice of the constants $c_{n}$ and divisibility constraints $d_{i j}$.

For every $n \in \mathbb{N}$ we choose $c_{n} \in \mathbb{N}$ such that

$$
c_{n} n \equiv 2^{n-1} \quad\left(\bmod 2^{n}\right) .
$$


To see that this is possible, write $n=2^{k} p$, where $p$ is odd and $k<n$. Then we seek $c_{n}$ such that

$$
c_{n} p \equiv 2^{n-k-1} \quad\left(\bmod 2^{n-k}\right) .
$$

But $p$ is odd, hence invertible mod $2^{n-k}$, so these congruences have solutions. For example, we can take $c_{1}=1, c_{2}=1, c_{3}=4, c_{4}=2$ and $c_{5}=16$.

We now show that the system (1) with this choice of coefficients provides a counterexample for Question 1.

Proposition 5. There is a 2-colouring of $\mathbb{N}$ such that there are no integers $y$ and $x_{i j}$ such that $x_{i j} \equiv 0\left(\bmod 2^{i}\right)$ and the system (1) is monochromatic.

Proof. Note first that all the variables must be positive, as they belong to the image, which is in $\mathbb{N}$. We shall define the colouring in stages, so that at the $n$th stage we force $n$ and the $n$th expression in the first column to have different colours. This rules out the possible values of $y$ one by one.

For $n=1$, we colour 1 red and all the other odd numbers blue. Since $x_{11}$ must be even and positive, and $c_{1}$ is odd, the first expression must be blue.

For $n=2$, we colour 2 red and all the other numbers that are $2 \bmod 4$ blue. Since $x_{21}$ and $x_{22}$ are $0 \bmod 4$ and positive, and $2 c_{2} \equiv 2(\bmod 4)$, the second expression must be blue.

For $n=3$, the number 3 has already been coloured blue. So we colour every number that is $4 \bmod 8$ red. Since $x_{31} \equiv x_{32} \equiv x_{33} \equiv 0(\bmod 8)$, and $3 c_{3} \equiv 4(\bmod 8)$, the third expression must be red.

Continue. At the $n$th stage, $n$ has already received some colour. Give all numbers which are $2^{n-1} \bmod 2^{n}$ the opposite colour to $n$. Since $x_{n 1} \equiv \cdots \equiv$ $x_{n n} \equiv 0\left(\bmod 2^{n}\right)$, and $c_{n} n \equiv 2^{n-1}\left(\bmod 2^{n}\right)$, the $n$th expression must have the opposite colour to $n$.

We now turn to Question 2. We will use a reformulation of Proposition 5 to obtain a counterexample. Consider the system below, obtained by reparameterising (1) by setting $x_{i j}=2^{i} z_{i j}$.

$$
\begin{array}{rcc}
2 z_{11}+c_{1} y & 2 z_{11} & y \\
4 z_{21}+4 z_{22}+c_{2} y & 4 z_{21} & \\
8 z_{31}+8 z_{32}+8 z_{33}+c_{3} y & 4 z_{22} & \\
\vdots & \vdots \\
2^{n} z_{n 1}+\cdots+2^{n} z_{n n}+c_{n} y & 2^{i} z_{i j} &
\end{array}
$$


The system (2) is not image partition regular, as if it were then taking $x_{i j}=2^{i} z_{i j}$ would contradict Proposition 5 . But if (1) is represented by the matrix $A_{1}$ and (2) is represented by the matrix $A_{2}$, then $B\left(A_{1}\right)=B\left(A_{2}\right)$, so image partition regularity of $A$ cannot be determined by examining the matrix $B(A)$. (In fact, $B\left(A_{1}\right)$ is kernel partition regular, which is the main result of [1].)

Finally, we observe that (1) and (2) have the same images over $\mathbb{Q}$. So $(2)$ is not image partition regular but is image partition regular over $\mathbb{Q}$, providing a counterexample for Question 3.

\section{References}

[1] B. Barber, N. Hindman, and I. Leader. Partition regularity in the rationals. J. Combinatorial Theory Ser. A. To appear.

[2] R. L. Graham, B. L. Rothschild, and J. H. Spencer (1990). Ramsey Theory, 2nd ed. Wiley-Interscience Series in Discrete Mathematics and Optimization. John Wiley \& Sons Inc., New York. MR1044995

[3] N. Hindman, I. Leader, and D. Strauss (2003). Open problems in partition regularity. Combin. Probab. Comput. 12 571-583. MR2037071

[4] N. Hindman and I. Leader (1993). Image partition regularity of matrices. Combin. Probab. Comput. 2(4) 437-463. MR1264718

[5] R. Rado (1933). Studien zur Kombinatorik. Math. Z. 36 424-470. MR1545354

BEN BARBER

Department of Pure Mathematics and Mathematical Statistics

Centre for Mathematical Sciences

WILBERFORCE ROAD

Cambridge, CB3 0WB

$\mathrm{UK}$

E-mail address: b.a.barber@dpmms.cam.ac.uk

IMRE LEADER

Department of Pure Mathematics and Mathematical Statistics

Centre for Mathematical Sciences

WILBERFORCE ROAD

Cambridge, CB3 0WB

UK

E-mail address: i.leader@dpmms.cam.ac.uk

ReCEIVED April 18, 2013 